\title{
Rinoplastia columelar: uma nova visão com uso de silicone sólido
}

\author{
Columellar rhinoplasty: a new vision using solid silicone
}

\begin{abstract}
José CARLOS Daher ${ }^{1}$
Marcela Caetano

CAMMAROTA $^{2}$

JEFFERSON Di LAMARTINE

GALDINO $^{2}$

Cesar Augusto Daher Ceva

FARIA $^{3}$

Alberto Benedik Neto 3

Gustavo De Souza

GuIMARÃES $^{4}$

\section{RESUMO}

Introdução: Nas rinoplastias, o tratamento do ângulo columelo-labial é ponto de relevância. A observação levou-nos a crer que, muitas vezes, o ângulo nasal deprimido e agudo ou a falta de projeção da ponta nasal devem-se a uma hipotrofia ou posição recuada do maxilar e da espinha nasal, tornando-os um suporte "recuado" para a base da pirâmide. Isto, associado à falta de sustentação ou reduzidas dimensões das cruras medialis das cartilagens alares, leva a narizes achatados, aduncos, podendo produzir pseudogibas. Objetivos: O objetivo deste trabalho é descrever uma nova concepção de rinoplastia, por técnica em que usamos uma pequena peça de silicone compacta, colocada por via de acesso no vestíbulo bucal, que se encaixará na columela e se apoiará na maxila e espinha nasal, funcionando como um verdadeiro avanço frontal, redefinindo a forma da columela e recolocando e redimensionando toda a base nasal, cuja ponta é projetada no espaço para frente. Método: $\mathrm{O}$ implante aloplástico foi esculpido em silicone "medium" (Silimed $\left.{ }^{\circledR}\right)$ ou "soft", com desenho prédefinido e que se ajustou a cada caso. O procedimento foi acompanhado (ou não) da fratura dos ossos nasais pelos processos convencionais. Resultados: Os resultados estéticos do procedimento são extremamente animadores, muitas vezes substituindo procedimentos de rinoplastias mais complexos e invasivos, ou complementando-os com eficiência. Em nossa prática de 32 anos de uso desta técnica, aplicamos o procedimento em 181 pacientes, com exposição do implante em apenas dois casos.
\end{abstract}

Trabalho realizado no Centro de

Estudos do Hospital Daher Lago Sul, Brasília, DF, Brasil.

Artigo submetido pelo SGP (Sistema de Gestão de Publicações) da RBCP.

Artigo recebido: 9/6/2010 Artigo aceito:18/8/2010

Descritores: Rinoplastia. Nariz/cirurgia. Próteses e implantes.

\section{SUMMARY}

Background: The treatment of columello labial angle is an important point in rhinoplasty. We believe that this angle is depressed and acute, in function of a retracted position of the maxilla and nasal spine, that represent a "back" support to the nasal pyramid basis. Objective: We describe in this paper our technique, using a small compact silicone device, included through a vestibular approach, introduced into the columella and supported in front of the maxilla, functioning like an actual advance of the maxilla, redefining the columella shape and projecting the all basis of nasal pyramid and consequently, the nasal tip. Methods: This alloplastic implant was carved in a "medium" or "soft" silicone block (Silimed( ${ }^{\circledR}$ ), following a pre defined design, adapted to each patient. Results: We have excellent aesthetic with this procedure, replacing many times a classic rhinoplasty, more complex and invasive procedure, or joining additional help associated to it. In our 32 years practice using this technique and among 181 treated patients, we had late expositions of this implant in only two cases.

Descriptors: Rhinoplasty. Nose/surgery. Prostheses and implants.

1. Membro titular da Sociedade Brasileira de Cirurgia Plástica (SBCP); Chefe do Serviço de Cirurgia Plástica do Hospital Daher Lago Sul, Brasília, DF.

2. Membro titular da SBCP; Cirurgião plástico do Serviço de Cirurgia Plástica do Hospital Daher Lago Sul, Brasília, DF.

3. Cirurgião geral pelo MEC, Residente do Serviço de Cirurgia Plástica do Hospital Daher Lago Sul, Brasília, DF.

4. Membro especialista da SBCP; colaborador do Serviço de Cirurgia Plástica do Hospital Daher Lago Sul, Brasília, DF. 


\section{INTRODUÇÃO}

A rinoplastia sempre foi um procedimento amplamente difundido e procurado, sendo muitas vezes ensinado e praticado visando exclusivamente, ou principalmente, os resultados estéticos, sem levar em consideração os aspectos funcionais da fisiologia respiratória. A técnica descrita por Joseph, em 1912, ainda hoje em uso por diversos cirurgiões com algumas alterações técnicas, era aplicada de maneira padronizada com a limitação da via de acesso das rinoplastias fechadas, limitando seus resultados.

Desaparecidos os edemas, que demoram às vezes anos, associados tardiamente a retrações cicatriciais tardiamente, desnudavam-se resultados frequentemente pobres e, muitas vezes, indesejáveis, com limitações funcionais, pelo menor cuidado com a preservação do revestimento interno do nariz e suas estruturas anatômicas, como as válvulas nasais.

Estas experiências levaram a procedimentos mais primorosos, extremamente evoluídos pelo farto campo operatório oferecido aos cirurgiões pela rinoplastia aberta (Rethi 1931).

Embora a rinoplastia aberta possa sugerir maior agressividade cirúrgica, pode também ser interpretada como via de acesso que permite movimentos mais precisos e conservadores, preservando ao máximo as estruturas e, sobretudo, complementando-as com os enxertos nasais quando necessários.

A partir deste período, o ângulo columelo-labial pôde ser tratado com mais exatidão, usando-se enxertos cartilaginosos ao invés de simplesmente encurtar-se o nariz como proposto por Joseph, em 1912.

É inegável que, apesar da enorme evolução contemporânea das rinoplastias, as técnicas abertas são mais invasivas, no entanto, existe uma preocupação infinitamente maior com a preservação da função respiratória, o que, de certa forma, foi menosprezado no passado, quando se valorizava mais os resultados estéticos imediatos.

Esta busca por melhores resultados associados a procedimentos menos invasivos, com a máxima preservação da função respiratória, levou-nos a procurar soluções mais simples, sem invalidar o uso das outras técnicas já consagradas.

Esta nova proposta de se "redesenhar" o ângulo columelo-labial com um pequeno implante de silicone foi apresentada inicialmente por Daher, em nota prévia no Congresso Brasileiro de Cirurgia Plástica, no Rio de Janeiro, em $1977^{19}$. Este procedimento tomou corpo em nossa prática médica à medida que se observavam os diversos efeitos benéficos que trazia também a outras estruturas que não o ângulo columelo-labial, tais como o afilamento da ponta e do nariz, a forma do triângulo base nasal e os efeitos sobre o dorso nasal. Terminou por se firmar como um procedimento próprio e alternativo de rinoplastia, que substitui a rinoplastia clássica em certos casos, ou a complementa em outros.

\section{MÉTODO}

Foi feito levantamento retrospectivo de nossos pacientes desde 1978, sendo recuperados os dados de 181 casos operados com esta técnica. Sendo selecionados os resultados de quatro pacientes para ilustrar este artigo.

Todas as peças implantadas foram esculpidas em bloco de silicone medium $\left(\right.$ Silimed $\left.^{\circledR}\right)$. As peças têm um desenho original (Figuras 1 a 4), com formato que pode ser descrito como uma Torre Eiffel estilizada, lapidada, com sua base em bloco retangular de $10 \mathrm{~mm}$ de largura, 3 a $5 \mathrm{~mm}$ na sua profundidade e $7 \mathrm{~mm}$ de altura, prolongando-se por uma coluna na forma da torre, com comprimento de $23 \mathrm{~mm}$ (perfazendo $30 \mathrm{~mm}$ de altura para toda a peça), sendo que sua haste mais fina terá um diâmetro de $2 \mathrm{~mm}$.

Este desenho básico foi reajustado pelo cirurgião, com bisturi, para cada caso, variando segundo as estruturas anatômicas e as demandas de cada paciente, conduzindo a vários outros modelos derivativos (Figuras 4 e 5). A haste que se encaixa na columela foi sempre apoiada no tecido conectivo inter crura medialis, não ultrapassando o ligamento interdomal, para que não se apoiasse diretamente na pele, e sua altura é facilmente ajustada de acordo com a projeção da ponta e ângulo columelo-labial desejados.

O pequeno bloco, base da peça, foi ajustado em suas dimensões, dependendo do quanto se desejava, ou não, acrescentar ao volume da pré-maxila propriamente dita, restando, às vezes, da peça praticamente uma haste. Em outros casos, foi esculpida uma nova peça com base de dimensões maiores que as descritas para maior projeção da base da columela e lábio superior.

\section{Técnica operatória}

A cirurgia pode ser realizada sob anestesia local, como procedimento único ou associada a outros procedimentos, sendo comumente associada nos pacientes de face lifting ou rinoplastias clássicas (nestes casos, a área columelar receptora deve estar intacta o bastante para ser confeccionada a loja receptora do implante, sem continuidade de outros descolamentos).

A via de acesso é o vestíbulo bucal, com incisão da mucosa em "V" invertido de aproximadamente $1 \mathrm{~cm}(0,5$ cm cada perna do "V"), bastante obtuso (próximo a $160^{\circ}$ ), que preserva o freio do lábio superior (Figura 6). Esta 


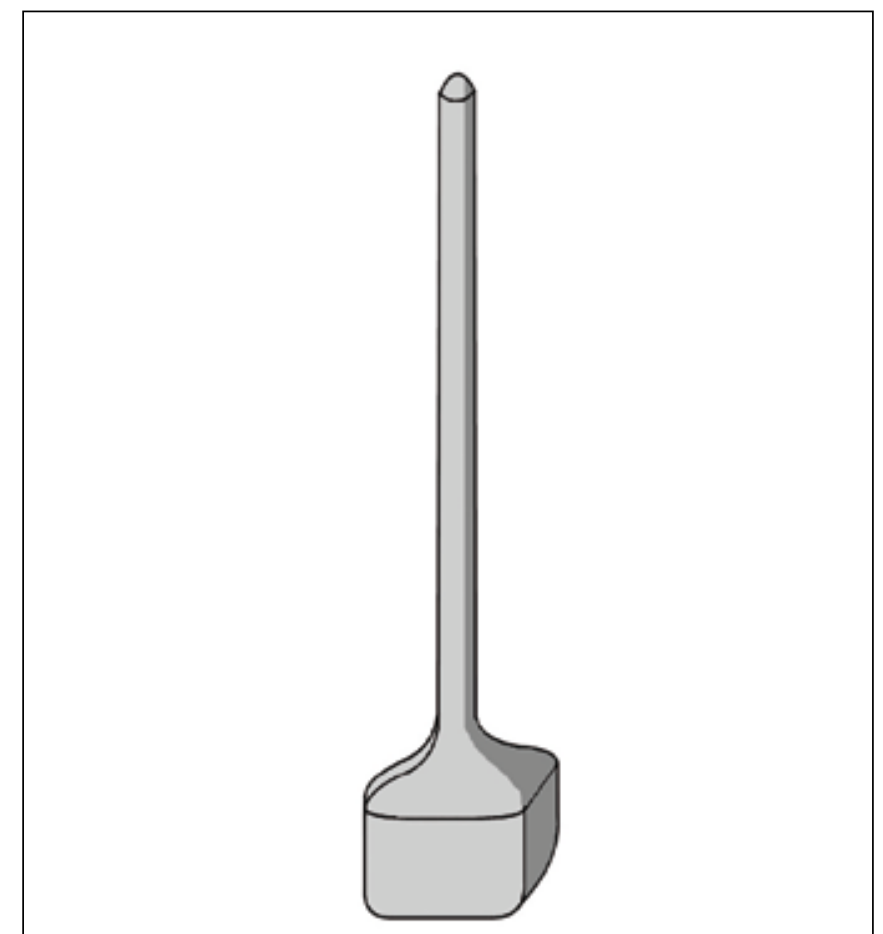

Figura 1 - Desenho da prótese de silicone sólido: vista em perspectiva.

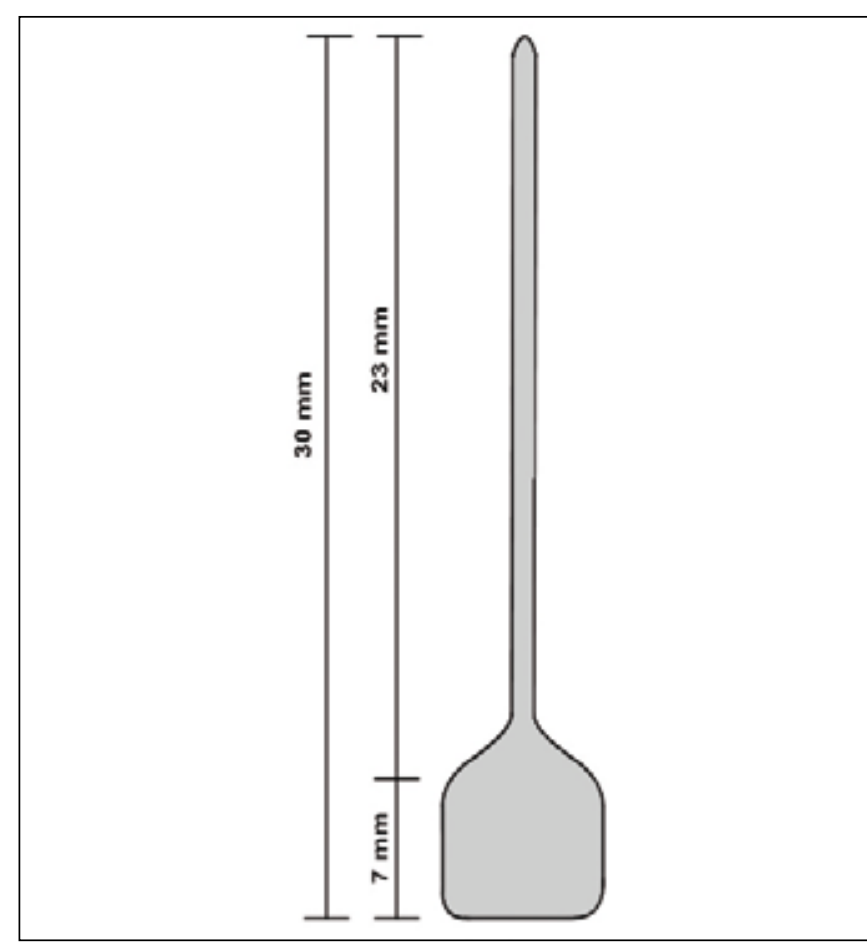

Figura 2 - Desenho demonstrando vista frontal da prótese e suas medidas.

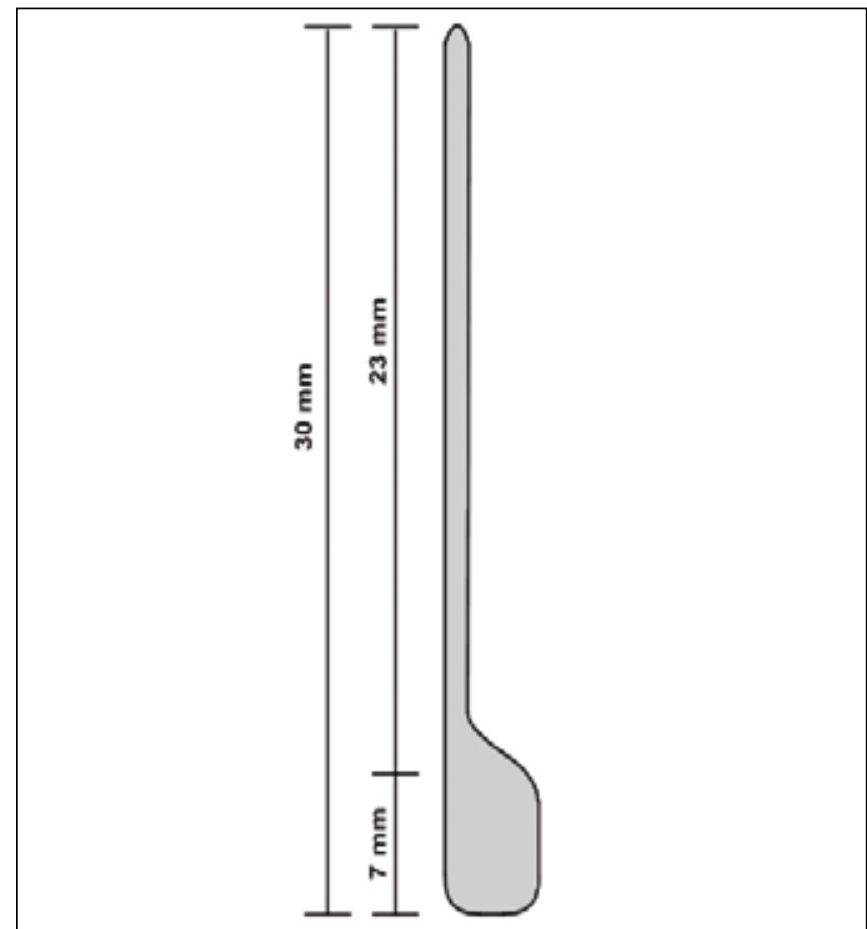

Figura 3 - Desenho demonstrando vista lateral da prótese e suas medidas.

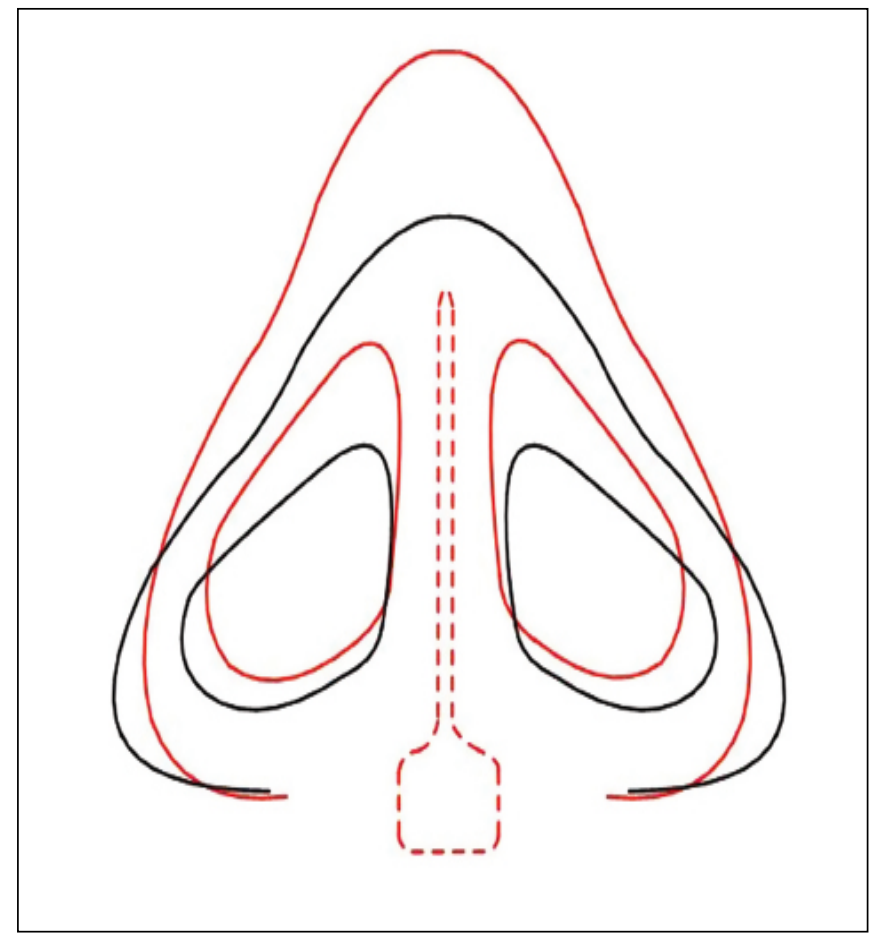

Figura 4 - Em preto, desenho de um nariz com baixa projeção da ponta e, em vermelho, o esboço das mudanças, as alterações pós-implante, demonstrando uma base nasal mais estreita e a ponta mais projetada. 


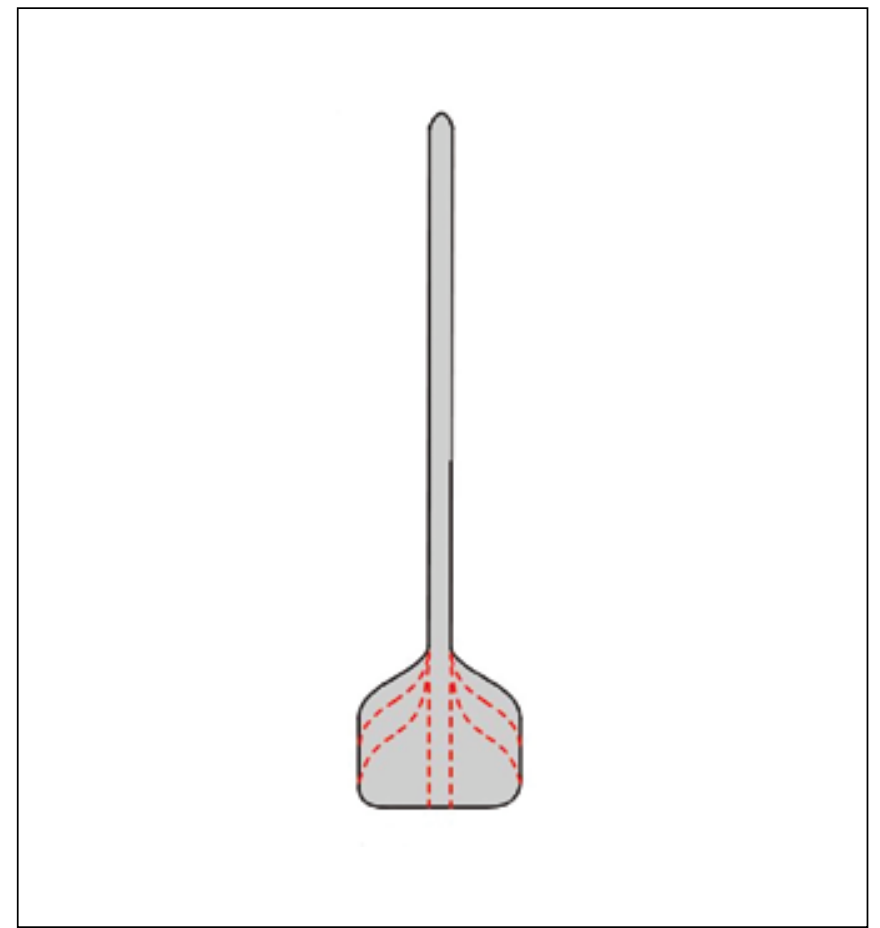

Figura 5 - Vista frontal da prótese com suas alternativas de modelagem intra-operatória, com bisturí.

incisão deve avançar através do plano muscular em direção à base da espinha nasal, confeccionando um retalho inferior espesso, de mucosa e músculo, que garantirá que o implante não ficará coberto apenas por mucosa à altura da reflexão no vestíbulo bucal.

Atingido o maxilar, com descolador de periósteo (Freer), se criará pequena loja descolando-se junto ao periósteo da maxila, onde se apoiará o implante, até se atingir a espinha nasal que será desnudada em sua face ventral (justo na dimensão para estar em contato com a base do implante). Esta dissecção continuará columela acima, sendo confeccionado espaço em seu interior produzido com a tesoura de Fomon. É fundamental que sejam preservados os tecidos de revestimento circunvizinhos a esta loja, a saber: mucosa do assoalho do seio piriforme, mucosa septal (septo mole) e a pele. Esta dissecção intracolumelar é indispensável para o perfeito encaixe da peça, e poderá prolongar-se segundo a necessidade de cada caso, porém jamais ultrapassando o limite do ligamento interdomal e jamais tocando a pele da ponta nasal.

Colocado o implante, são feitos os ajustes, retirando-se os excessos (o desenho original já é superdimensionado, oferecendo esta oportunidade ao cirurgião), até que se chegue a forma ideal (Figuras 7 e 8).

O implante será agora fixado ao periósteo da espinha

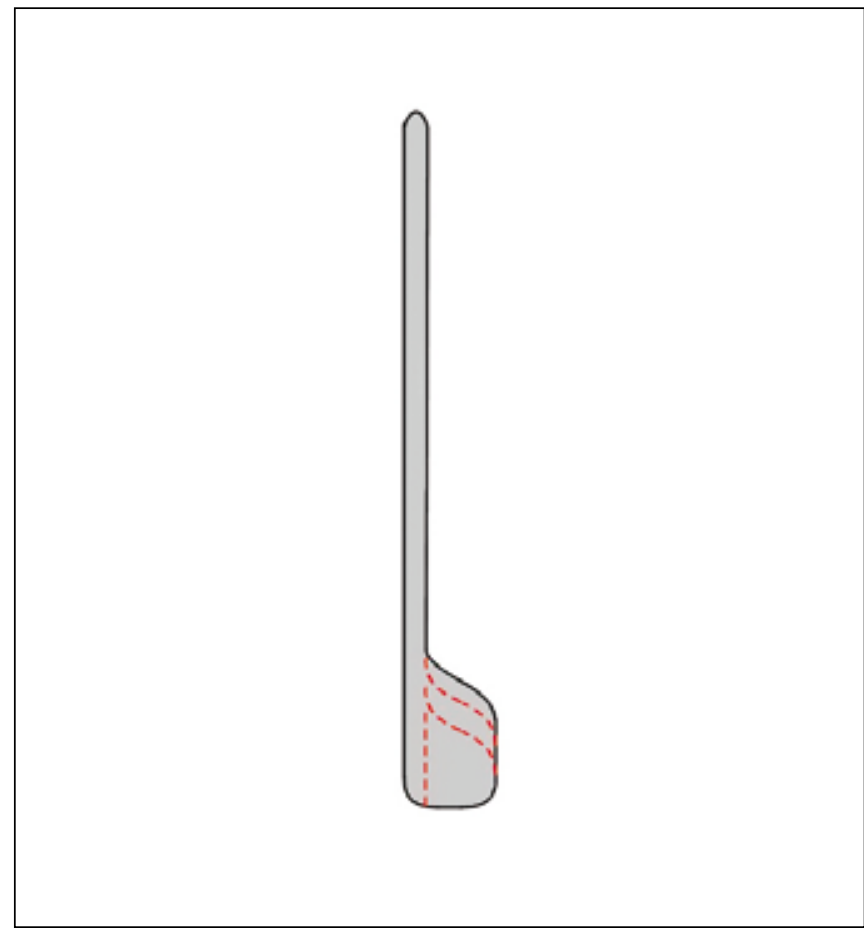

Figura 6 - Vista lateral da prótese com suas alternativas de modelagem intra-operatória, com bisturí.

nasal ou tecido do septo mole, imediatamente acima do seu ápice, por um único ponto de nylon 3-0.

A ferida operatória será fechada por planos, com um ponto em plano muscular com fio de nylon 4-0, trazendo as bordas deixadas na confecção da via de acesso (interessando o músculo depressor do septo e parte do orbicular dos lábios), e fechamento da mucosa com fio de catgut 4-0 (de 3 a 5 pontos simples).

Não há curativos externos para o procedimento especificamente, e usa-se antibioticoterapia por 7 dias.

\section{RESULTADOS}

Os resultados obtidos foram sempre satisfatórios dentro dos objetivos propostos: abrir o ângulo columelo-labial, originalmente agudo, e projetar a base columelar, bem como toda a base da pirâmide nasal, para frente. Este último efeito, considerando que a parte intracolumelar do implante está travada entre as cruras medialis, funcionará como um alongamento destas cruras, projetando o joelho das cartilagens alares, tornará o triângulo da base nasal de base mais estreita e mais afilado e esconderá, total ou parcialmente, a giba nasal pré-existente. Este procedimento poderá ser associado à fratura dos ossos nasais pelos procedimentos já classicamente descritos, quando se deseja estreitar o nariz em sua base (Figuras 9 a 12). 
Tivemos, em todo o período de uso desta técnica, dois casos de exposição dos implantes, que foram prontamente retirados. O primeiro caso atribuímos a uma lesão local na reflexão da mucosa vestibular, pois a paciente usava prótese dentária superior, de peça única e de encaixe, que traumatizava repetidamente a mucosa que ficava prensada

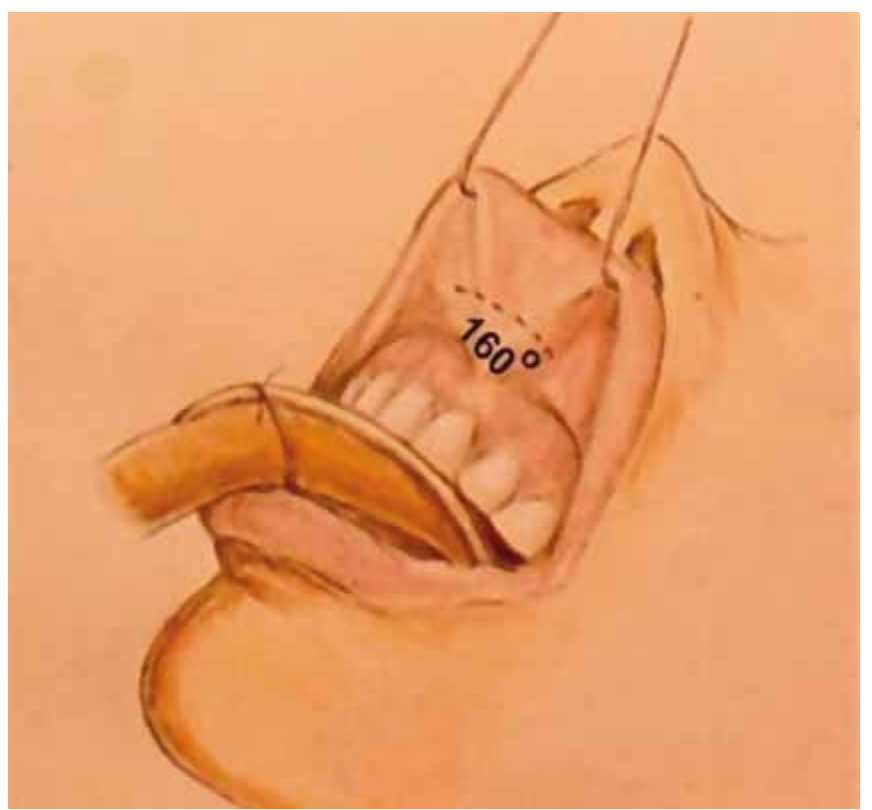

Figura 7 - Via de acesso pelo vestíbulo bucal, preservando o freio do lábio superior.

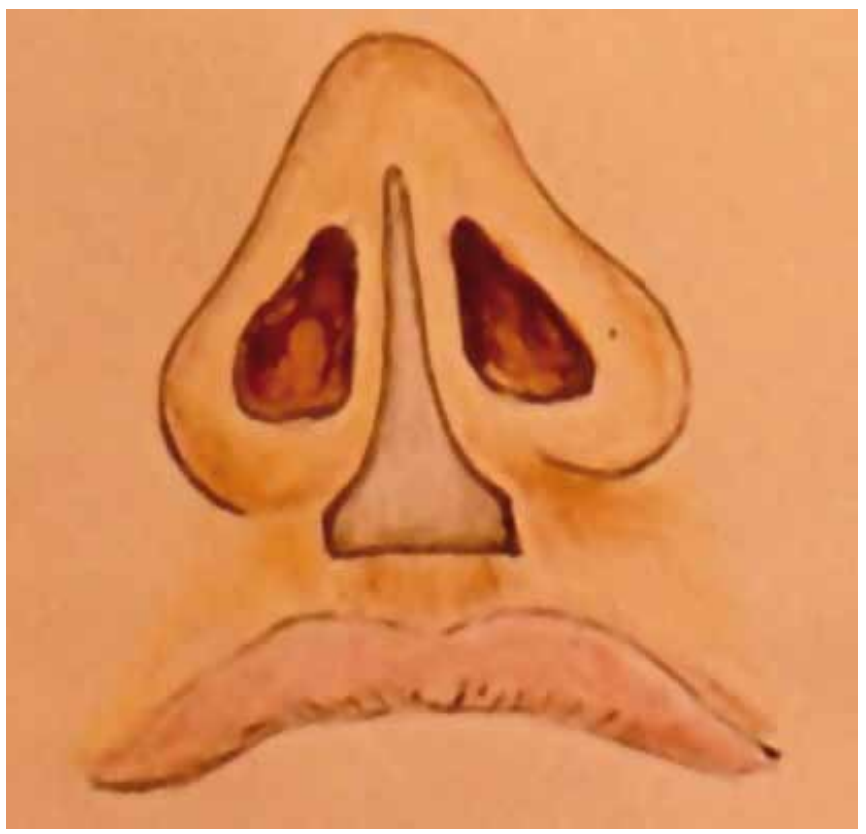

Figura 8 - Implante posicionado. entre a prótese e o implante. No segundo caso, houve lesão da mucosa no momento de confecção da bolsa. O orifício da lesão foi fechado com um ponto separado, mas evolui para exposição e infecção.

Dentre os casos iniciais, quando ainda não estava instituída a rotina de fixação de sua base na espinha nasal,
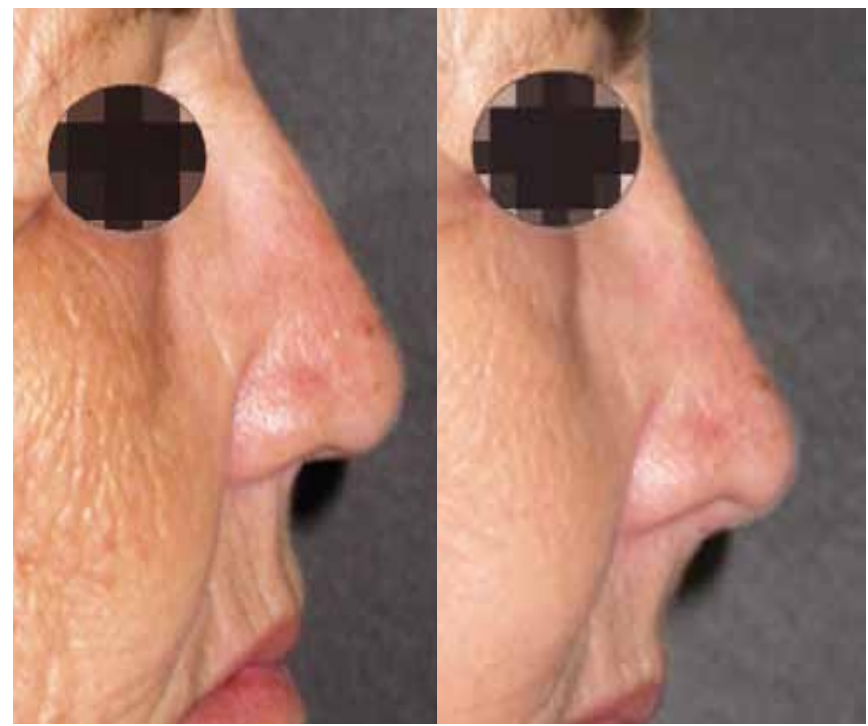

Figura 9 - Paciente I: Rinoplastia columelar associada a face lifting. Paciente de 69 anos com atrofia do maxilar. Observase, no pós-operatório, a projeção da ponta e consequente desaparecimento da giba com abertura do ângulo columelo-labial.

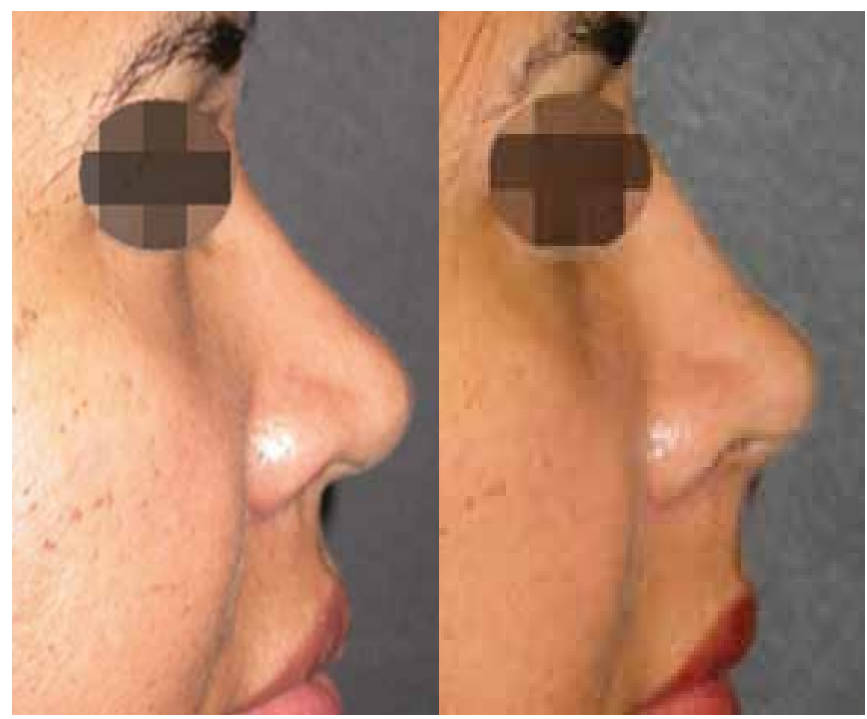

Figura 10 - Paciente II: Observa-se o efeito de avançamento da maxila, com melhor projeção da ponta, alongamento da columela e abertura do ângulo columelo-labial. Rinoplastia columelar simples sem necessidade de outros procedimentos. 


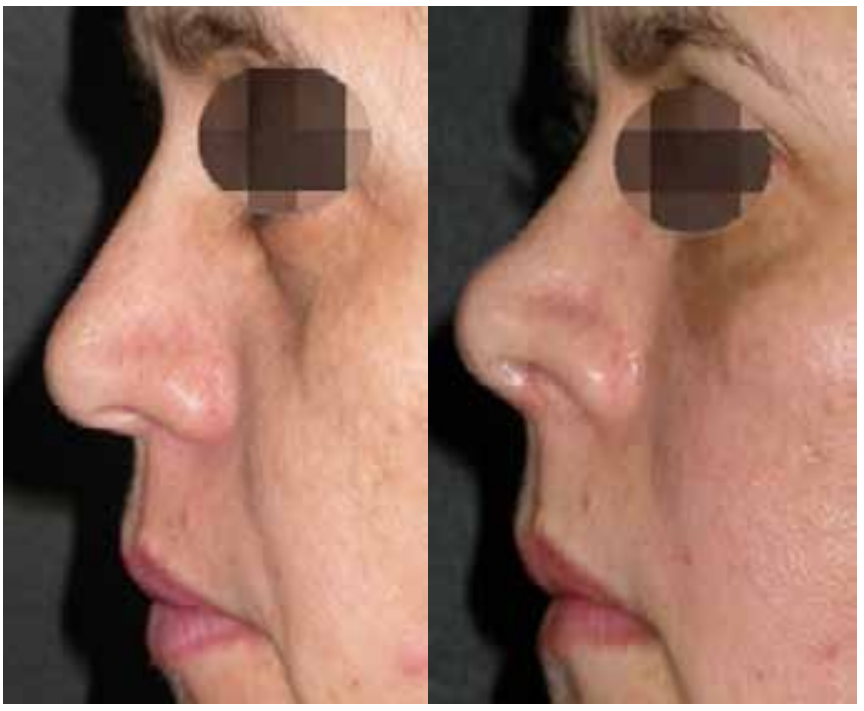

Figura 11 - Paciente III: Observa-se, no pré-operatório, um nariz de dorso longo, ligeiramente caído e ângulo columelolabial menor que 90 graus. No pós-operatório, nota-se a sensação de projeção do maxilar como um todo, com encurtamento do dorso, projeção da ponta, abertura do ângulo columelo-labial e verticalização do lábio superior.

houve dois casos de deslocamento de posição do implante; um deles foi reoperado para reposicionamento e o outro, sem reflexos estéticos, foi mantido por decisão da paciente.

\section{DISCUSSÃO}

Existe atualmente nos meios científicos um preconceito contra a utilização do silicone no dorso nasal. Embora existam opiniões de que maus resultados restrinjam-se a pacientes caucasianos ou de pele fina, o procedimento é amplamente divulgado nos países de raça amarela, principalmente no Japão. Em nosso serviço, estes implantes de silicone em dorso nasal sempre resultaram em exposição ou deslocamentos, sendo necessária sua retirada a longo $\operatorname{prazo}^{1-9,12-15}$.

Apesar de nossa posição restritiva ao uso do silicone no dorso nasal, mantivemos este procedimento para o ângulo columelo-labial, pela excelente relação custo-benefício, o que se mostrou, no decorrer de vários anos, perfeitamente exequível e seguro.

Muitas vezes, o simples avançamento da base da pirâmide nasal, por si só, representa grande melhora aos narizes desgraciosos, fazendo-os mais arrebitados, com pontas mais projetadas e, às vezes, com joelhos (domus) mais visíveis.

Nesta técnica, podemos constatar alguns fatos importantes: primeiramente, verificamos que há uma agudização

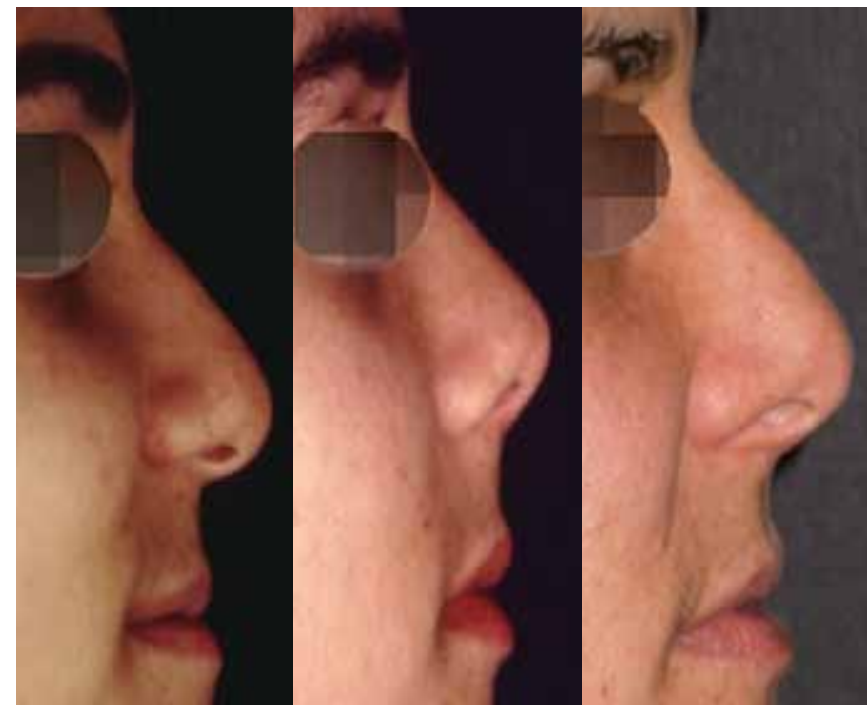

Figura 12 - Paciente IV: Paciente operada há 22 anos, aspectos pré-operatório, pós-operatório imediato e resultado tardio 22 anos após. Observa-se que houve projeção da ponta desfazendo o excesso de dorso e a duradoura remodelagem do ângulo columelolabial, que de agudo passa a obtuso. O resultado é duradouro, com perfeita adaptação e satisfação da paciente.

do ângulo formado entre as cruras medialis e lateralis das cartilagens alares, tornando joelho mais "agudo" e, portanto, mais proeminente sob a pele, marcando melhor sua presença e, às vezes, reproduzem com graciosidade os dois pontos luminosos da ponta nasal antes inexistentes. Este efeito é conseguido nas rinoplastias tradicionais, fechada ou aberta, pelos enxertos cartilaginosos. Além disso, a base nasal triangular, ao ter elevada sua ponta, ficará mais alta e acompanhando este movimento, sua base se estreitará, tornando o nariz mais afilado (Figura 8).

A elevação da ponta nasal, ou seja, sua projeção pósteroanterior no espaço, ajudará a esconder uma giba pré-existente, diminuindo-a ou eliminado-a totalmente. Assim, um nariz que no pré-operatório poderia supor a necessidade de ressecção de dorso, será resolvido pelo aumento de projeção da ponta. E ainda, se depois destas transformações a base nasal se mostrar larga, poderá ser associado o procedimento habitual de fratura dos ossos nasais, estreitando-se sua base.

É obvio que este tipo de rinoplastia, que rompe com todos os princípios clássicos, tem sua indicação restrita aos casos onde se podem vislumbrar vantagens com seus efeitos já bem definidos, e que são mais limitados em comparação às técnicas com amplas incisões, onde se pode "desmontar" toda a pirâmide nasal. Por outro lado, não se toca nas válvulas nasais e não se produz cicatrizes internas. Se, de certa forma isto é vantagem, representa também limitações 
para a correção de outras doenças, como as assimetrias das cartilagens alares, narizes pinçados e amplas septoplastias com repercussões externas. Isso reforça a necessidade de diagnóstico correto e indicação precisa para a técnica.

Considerando-se que o procedimento simples e pouco invasivo, pode ser facilmente associado a outras cirurgias de harmonização estética da face. Por exemplo, a melhora do ângulo columelo-labial e a projeção da ponta nasal são efeitos frequentemente desejáveis em pacientes de face lifting. Em busca deste objetivo, já se descreveu o descolamento do dorso nasal e sua tração via lifting frontal aberto ou usando muitos pequenos enxertos de cartilagem auricular, colhidos na incisão retroauricular do face lifting. Entretanto, nem sempre o face lifting está associado à abertura da região frontal e, em se tratando dos enxertos cartilaginosos, às vezes, o volume necessário para se produzir os efeitos estéticos desejados seria maior, sendo que o implante cartilaginoso colocado em grande quantidade junto ao maxilar frequentemente é reabsorvido, ainda que parcialmente. Este trabalho mostra exatamente uma alternativa onde não há reabsorção e a grande aceitação deste implante no corpo humano, além de ser um procedimento facilmente reversível.

Sugere-se que todos os pacientes submetidos a este procedimento, como em todos os outros de implante aloplástico, devem ser previamente esclarecidos da hipótese de eliminação, ainda que esta possibilidade, conforme este trabalho, se mostre comprovadamente remota.

Já se pretendeu obter os efeitos propostos pela técnica, com a inclusão de polimetilmetacrilato (PMMA). Os resultados demonstrados são similares aos que conseguimos, mas trazem em si toda a carga de discussões em torno destas inclusões ${ }^{10,11,16,17}$. Como alternativa para esta maior projeção da base da pirâmide nasal, estão os avançamentos de maxilar ${ }^{18}$ (tratamentos ortodônticos), ou o avançamento seletivo da espinha nasal e parte da maxila, com enxertos ósseos e fixação, procedimentos muito mais complexos e invasivos que o proposto.

Deve-se considerar como vantagem adicional do implante columelar de silicone sólido a ação antagônica que ele tem ao músculo depressor do septo nasal, neutralizando sua ação de tracionar para baixo a ponta nasal durante o sorriso, bem como mexer a ponta nasal durante a fala. Embora alguns autores manifestem preocupação quanto ao nariz "congelado", ainda acreditamos na franca opção dos pacientes por narizes bonitos, ainda que não mexam suas pontas.

Fator importante que deve ser observado e discutido especificamente com os pacientes é que o implante (em qualquer dimensão e principalmente quando é maior em sua base) pode alterar o desenho dos lábios durante o sorriso, visto que bloqueia parcialmente a natural elevação do lábio por sobre a gengiva em direção à espinha nasal, durante o sorriso.

Embora em certos pacientes este efeito seja desejável por diminuir a exposição do vermelhão, pode não ser por outros que prezam de maneira incondicional o sorriso que têm.

$\mathrm{O}$ mesmo cuidado deve-se ter com os pacientes que tocam instrumento de sopro, pois o procedimento pode modificar a embocadura, assim como qualquer procedimento que envolva a musculatura ou a relação anatômica dos lábios e estruturas vizinhas, inclusive nas rinoplastias clássicas.

Dentre todos os pontos discutidos, deve-se considerar também ser um procedimento perfeitamente reversível em caso de insatisfação com o resultado, seja por parte do paciente ou do cirurgião, o que nunca nos ocorreu com o uso desta técnica, até a presente data.

\section{CONCLUSÃO}

O uso do implante de silicone no ângulo columelo-labial na forma proposta pode ser considerado uma importante técnica alternativa de rinoplastia e é uma excelente ferramenta para o cirurgião plástico na harmonização da face. Consideramos ter excelente relação custo benefício pelos ótimos resultados e por ser simples, pouco invasivo, de resultados previsíveis, com baixíssimos índices de complicações, além de ser perfeitamente reversível.

\section{REFERÊNCIAS}

1. Lam SM. Revision rhinoplasty for the Asian nose. Facial Plast Surg. 2008;24(3):372-7.

2. Fedok FG. Revision rhinoplasty. Facial Plast Surg. 2008;24(3):269.

3. Jung DH, Kim BR, Choi JY, Rho YS, Park HJ, Han WW. Gross and pathologic analysis of long-term silicone implants inserted into the human body for augmentation rhinoplasty: 221 revision cases. Plast Reconstr Surg. 2007;120(7):1997-2003.

4. Adams JS. Grafts and implants in nasal and chin augmentation. A rational approach to material selection. Otolaryngol Clin North Am. 1987;20(4):913-30

5. Erlich MA, Parhiscar A. Nasal dorsal augmentation with silicone implants. Facial Plast Surg. 2003;19(4):325-30.

6. Deva AK, Merten S, Chang L. Silicone in nasal augmentation rhinoplasty: a decade of clinical experience. Plast Reconstr Surg. 1998;102(4):1230-7.

7. Yilmaz M, Vayvada H, Menderes A, Mola F, Atabey A. Dorsal nasal augmentation with rib cartilage graft: long-term results and patient satisfaction. J Craniofac Surg. 2007;18(6):1457-62.

8. Inanli S, Sari M, Baylancicek S. The use of expanded polytetrafluoroethylene (Gore-Tex) in rhinoplasty. Aesthetic Plast Surg. 2007;31(4):345-8.

9. Romo T 3rd, Kwak ES. Nasal grafts and implants in revision rhinoplasty. Facial Plast Surg Clin North Am. 2006;14(4):373-87. 
10. Berghaus A, Stelter K. Alloplastic materials in rhinoplasty. Curr Opin Otolaryngol Head Neck Surg. 2006;14(4):270-7.

11. Baran CN, Tiftikcioglu YO, Baran NK. The use of alloplastic materials in secondary rhinoplasties: 32 years of clinical experience. Plast Reconstr Surg. 2005;116(5):1502-16.

12. Tham C, Lai YL, Weng CJ, Chen YR. Silicone augmentation rhinoplasty in an Oriental population. Ann Plast Surg. 2005;54(1):1-7.

13. Perkins SW. The evolution of the combined use of endonasal and external columellar approaches to rhinoplasty. Facial Plast Surg Clin North Am. 2004;12(1):35-50.

14. Ahn J, Honrado C, Horn C. Combined silicone and cartilage implants: augmentation rhinoplasty in Asian patients. Arch Facial Plast Surg. 2004;6(2):120-3
15. Godin MS, Waldman SR, Johnson CM Jr. Nasal augmentation using GoreTex. A 10-year experience. Arch Facial Plast Surg. 1999;1(2):118-22.

16. Voldrich Z, Tománek Z, Vacík J, Kopecek JJ. Long-term experience with poly (glycol monomethacrylate) gel in plastic operations of the nose. Biomed Mater Res. 1975;9(6):675-85.

17. Lustica I, Velepic M, Cvjetković N, Bonifacić M, Kirincić N, Juretić M, et al. Polymethyl-methacrylate implants in forehead and supraorbital arches reconstruction: retrospective study. Coll Antropol. 2001;25(Suppl):137-43.

18. Hinderer UT. Nasal base, maxillary, and infraorbital implants: alloplastic. Clin Plast Surg. 1991;18(1):87-105.

19. Daher JC. Contribuição técnica às rinoplastias. XIV Congresso Brasileiro de Cirurgia Plástica;1977.

\section{Correspondência para:}

Cesar Augusto Daher Ceva Faria

SQSW 504 bloco K, 504 apto 506 - Sudoeste - Brasília, DF, Brasil - CEP 70673-511

E-mail: cesardaher@terra.com.br 